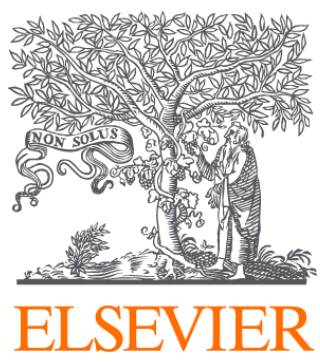

Since January 2020 Elsevier has created a COVID-19 resource centre with free information in English and Mandarin on the novel coronavirus COVID-

19. The COVID-19 resource centre is hosted on Elsevier Connect, the company's public news and information website.

Elsevier hereby grants permission to make all its COVID-19-related research that is available on the COVID-19 resource centre - including this research content - immediately available in PubMed Central and other publicly funded repositories, such as the WHO COVID database with rights for unrestricted research re-use and analyses in any form or by any means with acknowledgement of the original source. These permissions are granted for free by Elsevier for as long as the COVID-19 resource centre remains active. 


\title{
PEDIATRIC CLINICS
}

OF NORTH AMERICA

Pediatr Clin N Am 53 (2006) 195-214

\section{Pediatric Ear, Nose, and Throat Emergencies}

\author{
Morgen Bernius, $\mathrm{MD}^{\mathrm{a}, *}$, Donna Perlin, $\mathrm{MD}^{\mathrm{b}}$ \\ ${ }^{a}$ Department of Surgery, Division of Emergency Medicine, \\ University of Maryland School of Medicine, 110 Sout Paca Street, Sixth Floor, Suite 200, \\ Baltimore, MD 21201, USA \\ ${ }^{\mathrm{b}}$ Division of Pediatric Emergency Medicine, Department of Pediatrics, \\ University of Maryland Hospital for Children, 22 South Greene Street, Baltimore, MD 21201, USA
}

Acute otitis media $(\mathrm{AOM})$ is the most common infection for which antibiotics are prescribed in children, resulting in more than 20 million antibiotic prescriptions annually. New practice guidelines published by the American Academy of Pediatrics and the American Academy of Family Physicians [1] call for the judicious use of antibiotics in view of increasing antibiotic resistance and the unclear necessity of the use of antibiotics in children with uncomplicated AOM. These guidelines are reviewed below. In addition, this article reviews several other common ear, nose, and throat (ENT) entities, including sinusitis and dental emergencies, and current strategies in diagnosing and treating these conditions.

\section{Acute otitis externa}

Otitis externa is an inflammation of the external auditory canal and external surface of the tympanic membrane, which can be caused by a variety of conditions that compromise the lining of the canal. The ear canal is lined with squamous epithelial cells and cerumen glands that provide a lipid protective barrier [2]. When the skin of the ear canal is exposed to water for long periods, it becomes macerated and desquamates, causing the development of microfissures. The protective cerumen is washed away and the $\mathrm{pH}$ level of the ear canal changes [3]. Otitis externa is, therefore, seen frequently in swimmers, hot and humid climates, and in conditions that lead to water retention in the ear such as pro-

\footnotetext{
* Corresponding author.

E-mail address: morgen2131@aol.com (M. Bernius).
} 
longed showers. In addition, the skin of the canal can be disrupted by local trauma such as foreign bodies, cotton swabs, insect bites, and eczema. Other predisposing factors include hearing aids, earplugs, and an immunocompromised host.

Otitis externa is characterized by otalgia, ranging from an initial pruritus to severe pain, which is worse with motion or chewing. Pain is reproduced by pulling on the auricle or tragus. The canal becomes erythematous and increasingly swollen, leading to a sensation of aural fullness and possible hearing loss. Treatment should include cleansing and debridement of the ear canal with a cotton swab and hydrogen peroxide or Burow's solution. An acidifying agent ( $2 \%$ acetic acid) is useful to inhibit the growth of bacteria and fungi. Topical antibiotic drops alone or mixed with antifungal agents or steroids (to decrease inflammation) should be continued for at least 3 days after the resolution of symptoms. A cotton wick of gauze strip may be required to allow diffusion of the drops into the edematous ear canal. Nonsteroidal anti-inflammatory drugs (NSAIDs) or even opiates may be required for pain control. Cultures are not necessary unless the condition is refractory to treatment [4].

There are multiple types of otitis externa, including furunculosis (localized inflammation), diffuse bacterial otitis externa ("swimmer's ear"), otomycosis (fungal infection), and malignant or necrotizing otitis externa [4]. Each involves inflammation of the ear canal.

Furunculosis is a localized form of otitis externa, in which an abscess develops in a hair follicle with spreading of the infection to the surrounding skin. This usually occurs in the outer third of the ear canal. The most common causative agent is Staphylococcus aureus. Treatment includes the incision and drainage of pointing, fluctuant abscesses and the application of topical antistaphylococcal antibiotics.

Diffuse bacterial otitis externa, the most common type, is also known as swimmer's ear because of its most common predisposing factor. With prolonged exposure to water, the ear canal becomes colonized with gram-negative bacteria. Pseudomonas aeruginosa is the pathogen that most commonly causes diffuse bacterial otitis externa [4]. The initial symptom is usually pruritus that becomes increasingly painful as the infection progresses. Increasing erythema and edema of the auditory canal lead to a feeling of pressure and may lead to conductive hearing loss. Secretions may be scant serous to profuse and purulent. Systemic symptoms are usually absent.

Otomycosis, a chronic superficial infection of the ear canal, the tympanic membrane, or both is relatively rare and tends to occur in the tropics, in an immunocompromised host, or from prolonged topical steroid or antibiotic usage $[2,4]$. It may be primary or secondary. In primary fungal infection, the most common causative agent is Aspergillus organisms, which cause $80 \%$ to $90 \%$ of otomycosis infections [4]. It may also be caused by Candida organisms. The appearance of the debris in the canal can suggest a fungal infection because it has a peppered appearance secondary to black mycelial elements [4]. The most common presenting complaint with primary otomycosis is intense pruritus. Secondary otomycosis, which is a fungal infection superimposed on an existing 
bacterial infection, tends to present with intense pain. Treatment consists of applying topical antifungal agents such as clotrimazole or itraconazole [2], which may be combined with a topical steroid or an acidifying agent.

Malignant or necrotizing otitis externa is an advanced case of otitis externa presenting with tender preauricular lymphadenopathy, protrusion of a swollen auricle, and possible cellulitis of the skin over the mastoid bone [3]. There is severe erythema, edema, and tenderness of the ear canal and surrounding structures associated with intense pain and otorrhea. Facial nerve palsy may occasionally accompany malignant otitis externa, with possible involvement of other cranial nerves [4]. It is most commonly caused by $P$ aeruginosa and is often a complication of persistent otitis externa. Treatment requires systemic antibiotics, including oral ciprofloxacin, ofloxacin, or intravenous antipseudomonal drugs. Prolonged therapy is usually required [4]. Imaging with CT is often helpful to define the bone involvement and extent of disease, and follow-up CT scans are useful to follow the efficacy of treatment. ENT referral is recommended.

Prevention of otitis externa may be achieved by thoroughly drying the ear canal after bathing and swimming and instilling a $2 \%$ acetic acid solution or any of the over-the-counter swimmer's eardrops. The use of earplugs may also reduce infection in swimmers.

\section{Acute otitis media}

AOM is a closed-space inflammatory process in the middle ear, associated with a middle ear effusion (MEE), signs and symptoms of middle ear inflammation, and a recent, abrupt onset of clinical symptoms [1]. AOM is the most common infection for which antibiotics are prescribed in children, resulting in more than 20 million antibiotic prescriptions annually [1]. It has a prevalence that peaks at 6 to 20 months of age and has a propensity to become chronic and recurrent [5].

AOM must be differentiated from otitis media with effusion (OME), a nonsuppurative, secretory otitis media. Although OME may predispose to and may succeed AOM, it does not have an infectious component and, therefore, should be considered separately. AOM tends to occur with greater frequency in the first 2 years of life, possibly related to the immaturity of immunologic defenses and eustachian tube function and structure and perhaps to the greater proportions of time spent in the horizontal position [5]. It tends to occur more frequently in males and in Native American or Inuit populations [5]. It has been associated with lower socioeconomic status, possibly because of crowding, poor hygiene, suboptimal nutrition, and to limited access to health care and medical resources. Breastfeeding has been found to have a protective effect [5], whereas exposure to tobacco smoke and repeated exposure to other children, such as at a daycare facility, have been related to an increase in propensity toward the disease. Children who have craniofacial anomalies and Down syndrome have an increased prevalence of AOM [5]. 
The presence of a viral upper respiratory infection may lead to the development of AOM secondary to associated eustachian tube dysfunction, associated nasopharyngeal bacterial colonization, and damage to the respiratory tract epithelium with subsequent impairment of mucociliary clearance of bacteria [2] Pathogenic bacteria can be isolated from middle ear aspirates in approximately $70 \%$ of cases of AOM [5]. The three most common causative pathogens are Streptococcus pneumoniae, nontypable Haemophilus influenzae, and Moraxella catarrhalis [1,5]. A small number of cases involve group A streptococci, Staph aureus, and gram-negative organisms. Although viruses may be isolated from middle ear exudates, it is uncertain whether they can cause AOM without a bacterial co-pathogen [5].

The clinical presentation of AOM may be highly variable, especially in infants and toddlers. AOM may present with a history of an abrupt onset of otalgia, irritability, otorrhea, or fever. The presence of bulging of the tympanic membrane (TM) has the highest positive predictive value for the existence of MEE [1], followed by limited or absent mobility of the TM and air-fluid levels noted on otoscopy [1]. The TM may be erythematous or opacified.

The American Academy of Pediatrics and The American Academy of Family Physicians (AAP/AAFP) Clinical Practice Guideline specifies that the diagnosis of AOM meet all three requirements of rapid onset, the presence of MEE, and the presence of signs and symptoms of middle ear inflammation [1]. These same guidelines call for the judicious use of antibiotics in view of increasing antibiotic resistance and the unclear necessity of the use of antibiotics in children with uncomplicated AOM. Guidelines advocate the option of a period of observation without the use of antimicrobial agents for 48 to 72 hours in selected children with uncomplicated AOM. These cases include children from 6 months to 2 years of age with nonsevere illness if the diagnosis of AOM is uncertain and children older than 2 years of age with nonsevere illness, despite the certainty of the diagnosis of AOM. If the patient fails to improve with symptomatic treatment within 48 to 72 hours, the guidelines recommend that the patient be reassessed and antibiotic therapy initiated [1].

A recent study from the United Kingdom [6] compared a group of children aged 6 months to 10 years who received the observation option with a similar group of children who received immediate antibiotics. The authors found that $76 \%$ of children in the observation group never required antimicrobials. The immediate use of antibiotics was associated with a 1-day reduction of illness but no difference in school absence or pain scores.

If the decision is made to treat AOM with antibiotics, amoxicillin is recommended by the AAP/AAFP guidelines at a dosage of 80 to $90 \mathrm{mg} / \mathrm{kg} / \mathrm{d}$ [1]. Patients who are allergic to penicillin may alternatively be treated with azithromycin, clarithromycin, or erythromycin-sulfisoxazole. A course of 10 days is recommended for children younger than 6 year of age [7]. If the illness is severe or amoxicillin treatment has failed, amoxicillin-clavulanate, $90 \mathrm{mg} / \mathrm{kg} / \mathrm{d}$, would be the antimicrobial of choice to increase the coverage for $\beta$-lactamaseproducing $H$ influenzae and $M$ catarrhalis [8]. 
In the patient who is vomiting, a single dose of ceftriaxone, $50 \mathrm{mg} / \mathrm{kg}$, given intramuscularly, has been shown to be effective in the treatment of AOM $[9,10]$. The patient who has failed amoxicillin-clavulanate therapy may be considered for treatment with a 3-day course of parenteral ceftriaxone [10] or, alternatively, a course of oral clindamycin [1].

\section{Foreign bodies of the nose and ear}

Foreign bodies are a relatively common occurrence in the pediatric population. A wide variety of objects have been retrieved from both the ear and nose, including toys, food, paper, beads, and insects. The child may present with a clear history of foreign body self-insertion or, alternatively, may present with recurrent epistaxis, foul odor, or pain in the ear or nose. Impairment in hearing [11] or olfaction may be the presenting complaint. Many children are asymptomatic, and the foreign body is discovered by a parent or a physician during a routine examination.

Physical findings may vary with the duration of the foreign body impaction, the size of the foreign body, and the physical characteristics of the foreign body itself. In the case of a small object that has been inserted very recently, the child may be asymptomatic, whereas with a large foreign body, the child may present with evidence of local trauma. An insect in the ear canal often presents with intense, constant pain and a sensation of movement in the ear. A child with a chronic foreign body may present with unilateral nasal or otic discharge, a foul odor, or recurrent epistaxis secondary to chronic irritation [12].

Special mention should be made of battery impactions. Children can easily insert small batteries from electronic devices (eg, calculators, handheld video games, or watches) into their nostrils. Button batteries may release small amounts of chemicals and voltage that may lead to alkaline chemical burns, necrosis, or septal-tympanic perforation [13]. Batteries should be removed as soon as possible to prevent these complications [12].

The removal of nasal or otic foreign bodies should only be attempted in the emergency department if there is reasonable expectation for removal because repeated, failed attempts result in increased swelling and trauma and possibly repositioning the object into a less favorable location [12]. The child needs to be restrained adequately or sedated, and all necessary supplies should be readily available, including a good light source, nasal speculum, alligator forceps, a curette, suction apparatus, and a topical vasoconstrictor to reduce tissue edema before attempt at removal [12]. Foreign body removal may be accomplished by manually grasping the object with forceps, getting behind the object with a curette or irrigation, or by applying suction to the surface of the foreign body.

The physical characteristics of the foreign body as well as the anatomic location should be considered before removal is attempted [12]. A live insect will be most easily removed if it is first killed with either $2 \%$ lidocaine or mineral oil. Irrigation should be avoided with objects such as vegetable matter or sponges 
because the added water will make the foreign body swell, impeding its extraction [12]. A smooth round foreign body that completely occludes the orifice is not likely to be removed successfully with a curette or forceps and may benefit from suction. Positive pressure ventilation with either a bag-valve mask or mouthto-mouth pressure from the caregiver while occluding the noninvolved nostril has been successful in the removal of large, occlusive nasal foreign bodies [14]. Foreign bodies that are unable to be removed in the emergency department should be referred to an otolaryngologist for removal. Oral antibiotics are recommended in this situation to prevent infection of the obstructed orifice [12].

\section{Epistaxis}

The nose has a rich vascular supply, making it vulnerable to episodes of bleeding, either spontaneously or as a result of localized trauma. Most of the time, the bleeding is mild and self-limited, although it can be profuse and life threatening. In the pediatric population, epistaxis is most commonly the result of local trauma from nose picking or a recent upper respiratory infection [15]. Other causes include facial trauma, foreign bodies, cocaine or heroin sniffing, or sinusitis. Less commonly, systemic causes such as hepatic disease, leukemia, idiopathic thrombocytopenia, or coagulopathies may be associated with epistaxis. Coagulopathies may be congenital, such as von Willebrand's disease, hemophilia, and Osler-Weber-Rendu disease, or acquired (NSAIDs, aspirin, and rat poison) [15].

Epistaxis can be classified according to location of bleeding in the nasal cavity as either anterior or posterior. Anterior bleeding accounts for approximately $90 \%$ of episodes of epistaxis [16] and usually arises from a venous vascular plexus on the anterior nasal septum, known as Kiesselbach's plexus. The mucosa overlying Kiesselbach's plexus is fragile and firmly adhered to the cartilage of the septum, making it prone to trauma. Because the bleeding is mostly from capillary or venous sources, anterior bleeds tend to be characterized by a slow, persistent oozing [16]. Posterior bleeds, however, originate from the sphenopalatine artery in the posterior nasal cavity and nasopharynx [17] and tend to bleed more profusely. This type of bleeding carries a higher risk of airway compromise, aspiration of blood, and life-threatening hemorrhage [16].

Epistaxis tends to occur with a bimodal incidence [17]. Episodes that occur in the 2- to 10-year-old category are more frequently minor anterior bleeds, whereas bleeding that occurs in the second peak of those over the age 50 years is more likely to be more severe posterior bleeding [15]. Most cases of epistaxis require minimal intervention. Mild epistaxis without active bleeding requires only recommendations to minimize the recurrence of bleeding, including limiting local trauma (eg, no nose blowing or picking) and applying nasal hydration with saline mist or ointments and increasing ambient humidity with a cool mist vaporizer to reduce mucosal irritation.

If minor bleeding recurs, the patient (or parent) may be instructed to pinch the nostrils together for 5 to 30 minutes nonstop (without frequent peeking to see if 
the bleeding is controlled) and to keep the head elevated but not hyperextended to avoid aspiration of the blood [2]. Usually only 5 to 10 minutes is required to stop the bleeding. A piece of gauze soaked with a nasal decongestant spray, epinephrine at a ratio of 1:10,000, or phenylephrine and placed in the affected nostril may also be helpful to induce localized vasoconstriction. If bleeding cannot be controlled with these simple measures, then transfer to an emergency department is required.

The emergency department initial evaluation of the patient with epistaxis involves the assessment of the airway, breathing, and circulation (ABC). A posterior bleed is more likely than an anterior bleed to cause airway compromise or blood aspiration and may rarely require intubation to protect the airway. Hemodynamic instability must be evaluated and quickly addressed. Once cardiopulmonary stability has been established, the physical examination should include a nasal examination with a good light source and a handheld nasal speculum. The nasal cavity should first be cleared of clots with suction and forceps to allow visualization of the nasal mucosa and evaluation of obvious sources of bleeding [15].

If an anterior source of bleeding is identified and the bleeding is active, cautery may be helpful after localized anesthesia and topical vasoconstriction, using either silver nitrate sticks or thermal cautery. Cautery causes injury to the nasal mucosa, resulting in coagulation or blood vessel constriction to effect cessation of the bleeding [15]. Care must be taken when cauterizing a bleed to prevent damage to surrounding structures and perforation of the nasal septum. Anterior bleeding that is unresponsive to cautery may require anterior nasal packing with petroleum jelly (Vasoline) gauze strips or a commercial packing that expands when it becomes wet to tamponade the bleeding vessel. Packing should be preceded by the application of local anesthesia and nasal decongestants [15]. The removal of packing and a reevaluation can take place after 15 to 30 minutes.

The failure to identify an anterior source of bleeding is a possible indication of posterior bleeding. Posterior bleeding may be indicated by hemoptysis, hematemesis, or blood in the posterior pharynx [17]. Posterior epistaxis is less amenable to cautery secondary to the quantity of bleeding and often requires posterior nasal packing or epistaxis balloons [15]. These patients should be managed in conjunction with a specialist and usually require admission to the hospital because of the risk of hypoxia or respiratory compromise. In addition, children with a posterior nasal packing or epistaxis balloon should be treated with a course of antibiotics that provides staphylococcal and streptococcal coverage to decrease the incidence of sinusitis or toxic shock syndrome.

\section{Sinusitis}

Sinusitis is a relatively common form of upper respiratory tract infection in the pediatric population. The challenge for the emergency pediatric physician is to distinguish among an uncomplicated viral or allergic rhinosinusitis and acute, 
subacute, and chronic bacterial sinusitis. The classic presenting symptoms of sinusitis in adults and teenage patients with mature sinuses are rarely present in the preadolescent patient.

Sinus development starts in utero and continues through the teenage years. The maxillary and ethmoid sinuses form during the third to fourth months of gestation and are the only sinuses present at birth. The frontal sinus begins to develop by 1 to 2 years of age from an anterior ethmoid cell and migrates to a supraorbital position. The frontal sinus is not seen radiologically until approximately age 5 or 6 . By the early teenage years, the sinuses have enlarged to their adult capacity.

The maxillary, frontal, and anterior ethmoid sinuses drain through ostia into the middle meatus, beneath the middle turbinate in the nose. The outflow tract of the maxillary sinus is located on the upper medial wall of the sinus, which makes drainage by gravity difficult and predisposes patients to infection. The ethmoid sinuses are composed of multiple individual air cells that each drain through their own narrow ostia. These narrow tracts may be obstructed by thick secretions or mucosal inflammation, resulting in infection of the ethmoid sinuses. The frontal and sphenoid sinuses are, less commonly, primary sites of infection; however, they may be affected in pansinusitis, and infection may spread from them to the orbit or central nervous system.

\section{Acute bacterial sinusitis}

The classic presenting symptoms for acute bacterial sinusitis in the adult population (including fever, headache, facial pain, tenderness, and facial edema) are less common in children. Fever, if present, is often low grade. Nasal discharge is a common symptom, but the quality of nasal discharge varies from thin and clear to thick and purulent. Patients often have cough, although it is not always described as productive. Cough may be present in the daytime, although it is often worse at night or when lying supinely during naps.

In the pediatric patient, two qualities can help distinguish between a viral rhinosinusitis and an acute bacterial sinusitis:

- Persistence of symptoms: Upper respiratory tract infections with symptoms that last more than 10 days, without any improvement, are more likely to represent bacterial sinusitis [18]. Most uncomplicated viral infections last approximately 5 to 7 days, and if not resolved by the tenth day, they have at least shown significant improvement.

- Increased severity of symptoms: Severe symptoms are defined as the combination of high fever $\left(\geq 39^{\circ} \mathrm{C}\left[\geq 102^{\circ} \mathrm{F}\right]\right)$ and purulent nasal discharge or classic symptoms of acute sinusitis such as facial swelling and pain $[18,19]$. The presence of high fever and purulent nasal discharge lasting for at least 3 to 4 days indicate a likely bacterial sinusitis. Patients with viral nasosinusitis may have fever, but it is usually associated with constitutional 
symptoms in the early or prodromal phase of illness. Purulent nasal discharge may also develop in patients with viral infections, but it usually occurs later in the course of illness and clears to a watery or mucoid consistency before resolution.

\section{Subacute and chronic bacterial sinusitis}

The duration of symptoms of more than 30 days separates acute from subacute or chronic bacterial sinusitis. Nasal congestion (as opposed to discharge) and cough are the most common respiratory symptoms. Sore throat may be present from constant mouth breathing. Headache is a less common symptom, and fever is extremely rare [18].

\section{Diagnostic imaging}

Plain radiographs, including the Waters (occipitofrontal), Caldwell (angled posteroanterior), and lateral views, have traditionally been used to aid in diagnosing sinusitis. Radiographic findings that suggest a diagnosis of sinusitis (in the presence of appropriate clinical symptoms) include diffuse opacification, mucosal thickening ( $\geq 4 \mathrm{~mm}$ ), or air-fluid levels [20]. CT scanning of the sinuses has been the gold standard for diagnosis. Because of the speed and increased accessibility of CT scanners in the emergency department, sinus CT scanning is becoming the first-line choice for radiographic diagnosis of sinusitis.

\section{Treatment}

Sinusitis does have a high spontaneous cure rate, but antibiotics remain a mainstay of treatment in any pediatric practice [21]. The first-line therapy for uncomplicated acute bacterial sinusitis is high-dose amoxicillin, although with emerging resistance patterns in certain areas, there are a number of other therapeutic options. Recommendations on the choice of antibiotic therapy are similar to those for otitis media infection.

Initial treatment with an antibiotic with broader coverage, such as amoxicillinclavulanate or cefuroxime, is warranted in patients who show no improvement on amoxicillin or who have been treated with amoxicillin within the previous month, in locations with a high prevalence of $\beta$-lactamase-producing H influenzae, in the presence of frontal or sphenoid sinusitis or complicated ethmoidal sinusitis, or with a diagnosis of subacute or chronic sinusitis. In areas with a high frequency of infection with penicillin- and cephalosporin-resistant pneumococci, other antibiotics are available, including azithromycin, trimethoprim-sulfamethoxazole, and clindamycin. Antibiotic choices should be guided by culture and sensitivities when available. The duration of treatment is usually 10 to 14 days. Most patients 
show improvement within 3 to 4 days, and the guidelines recommendation continuing treatment for 7 days after the patient is asymptomatic [18].

\section{Oral cavity lesions}

\section{Herpes simplex virus}

The most common presentation of primary herpes simplex virus (HSV) infection in young children is herpetic gingivostomatitis. It is seen most often in children ages 6 months to 5 years. Because of its significant discomfort and disturbing appearance, it regularly triggers physician and emergency department visits. HSV is transmitted through infectious saliva and has an incubation period of 2 to 12 days, with a mean of 4 days. Viral shedding, however, can occur from 7 days to as long as 3 weeks or more after clinical infection [22]. The primary infection may present with associated flu-like symptoms, including an abrupt onset of high fever, irritability, and malaise. Oral findings include erythematous, edematous, and friable gingivae as well as oral and perioral clusters of vesicles, which coalesce to form large, painful ulcers. Symptoms usually last less than 1 week but may continue for up to 21 days [23].

HSV is usually diagnosed clinically, although confirmation is possible by viral culture of the vesicular fluid or by the identification of multinucleated giant cells on a Tzanck smear from the base of the lesions. The treatment of herpetic gingivostomatitis is primarily supportive. Analgesics and antipyretics (such as acetaminophen and ibuprofen) can be used. In more severe cases, oral opiates may be necessary. Topical anesthetics such as diphenhydramine syrup mixed in a 1:1 solution with magnesia hydroxide (Maalox) may be applied to the affected mucosa every 2 hours [24]. A small amount of the solution may be painted on the lesions of younger children. Older children may be instructed to spit out the solution after rinsing but should not swallow it. Although viscous lidocaine is commonly included in this topical solution, it should be avoided because of its potential for toxicity. Other nonprescription medications that can be used topically as a barrier to irritants include zilactin, docosanol (Abreva), and ORA-5, although their use is off-label in children [24].

Because of the significant pain associated with HSV lesions, children are at risk of dehydration from decreased oral intake. Oral fluid administration should be highly encouraged. Parents should be advised to give children oral fluids in the form of popsicles or ice, which soothe the oral lesions and are therefore more readily consumed.

Antiviral therapy may be helpful for the treatment of oral HSV disease if it is started within 72 hours of the onset of lesions [25]. Patients treated early with acyclovir have a shorter duration of lesions, earlier resolution of fever and extraoral lesions, a shorter duration of poor oral intake, and decreased viral shedding [26]. Topical acyclovir has no role in the treatment of primary oral HSV 
infection $[25,27]$. Although patients may be at risk for secondary bacterial infection, routine prophylactic antibiotic therapy is not indicated.

\section{Coxsackievirus}

Oropharyngeal infection with coxsackieviruses A and B manifest as herpangina and usually occurs in children 3 to 10 years of age. Patients present with painful vesicles and ulcerations of the posterior pharynx and tonsils, accompanied by flu-like symptoms that include fever, sore throat, and headache and occasionally vomiting and abdominal pain. The vesicles rupture, leaving fibrincovered ulcerations, which classically appear as grayish-white-based ulcers with erythematous borders. Herpangina is highly contagious, but the disease is self-limited, with symptoms usually resolving within 3 to 7 days. Treatment is symptomatic and consists of oral and topical analgesics.

Skin infected with the coxsackievirus A manifests as hand, foot, and mouth (HFM) disease. HFM disease most often affects children under the age of 5 . It presents with fever, painful cutaneous lesions on the dorsum of the hands and feet (and less often the buttocks and genitalia), and vesicles and ulcerations of the palate, tongue, and buccal mucosa. HFM disease is also self-limited, with symptoms usually resolving within 2 to 3 days. Treatment consists of analgesics and reassurance.

\section{Dental trauma}

Dental trauma occurs commonly in childhood. If they are not managed properly, these injuries can have significant long-term functional, cosmetic, and psychosocial effects. The emergency physician must be familiar with the appropriate management and referral needs for patients with dental injuries because outcomes are most often determined by the timeliness of intervention.

Most pediatric dental injuries occur in the toddler years as children become ambulatory [28]. Falls are the most common cause of dental injury in preschool and school-aged children, whereas sports-related injuries are more common in adolescents [29]. The maxillary central incisors are injured most commonly, followed by the maxillary lateral incisors and the mandibular incisors [30].

The possibility of abuse in any pediatric trauma patient must always be considered. Orofacial injuries may occur in up to $75 \%$ of abused children, so a high index of suspicion must be maintained when evaluating such patients [31]. Factors such as delay in seeking medical attention, inconsistent, unavailable, or changing history of the injury, torn upper labial frenula (except in the child who is learning to walk), bruising of the labial sulcus (in patients who are not walking) and the soft tissues of the cheek or neck (as opposed to the forehead or chin), inappropriate affect of the parent or patient, a history of past injuries, and wounds at varying stages of healing should arouse suspicion for abuse or neglect [32]. 


\section{Dental anatomy}

Each tooth consists of an external crown and one or more internal roots (Fig. 1). The crown consists of a hard outer layer of enamel, which protects both the inner layer of dentin and the small portion of the pulp that extends into the crown. The pulp is the vascular soft tissue component of the tooth that acts to form and provide nourishment to the dentin and to provide sensation to the tooth. The root extends into the alveolar bone and is covered by a layer of bone-like cementum. Individual sockets within the maxillary and mandibular alveolar bone house each tooth. The root is attached to the socket through the thin fibrous periodontal ligament, which in turn acts as a cushion between the tooth and bone to prevent trauma during mastication. The tip of each root is called the apex of the tooth and is the site of the neurovascular foramen.

\section{History and physical examination}

One of the most important historical factors in evaluating the patient with dental trauma is the time of injury because it affects both treatment and prognosis. The age of the patient and whether the injury is to primary (as opposed to permanent) teeth will also affect management decisions. Understanding the mechanism of injury helps determine both the severity of dental trauma and the likelihood of associated injuries. The patient's tetanus status should also be determined.

Complaints that may indicate a higher severity of injury and thus require more urgent dental referral include spontaneous tooth pain or temperature sensitivity, which may result from pulpal exposure or inflammation, pain with touch or pressure, which may indicate periodontal ligament damage, and dental malocclusion, which may result from a facial fracture or a displaced tooth.

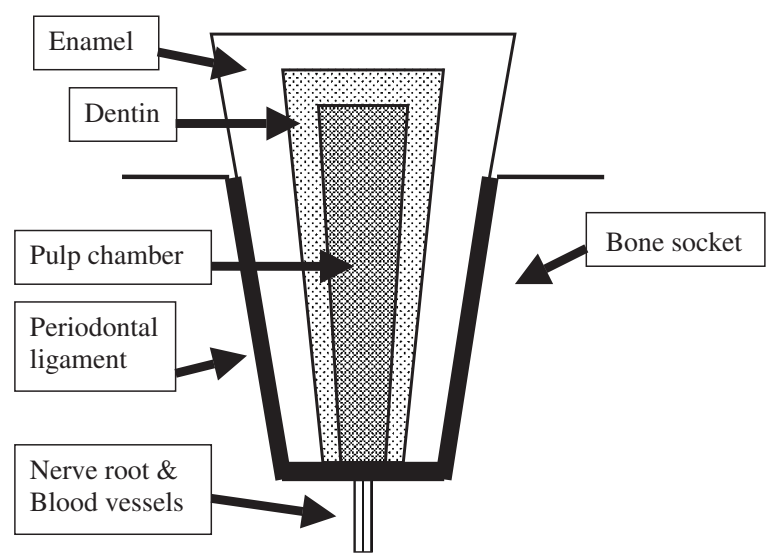

Fig. 1. Dental anatomy. 
Physical examination is guided by the mechanism of trauma and suspicion for associated injuries. A thorough neurologic examination should always be performed because dental injuries are a subset of head trauma. The most important aspects of the focused dental examination include palpation, percussion, and mobility evaluation. Adjacent soft tissues should be evaluated for injury and embedded tooth fragments.

\section{Radiographic examination}

Children with dental fractures, luxation, tooth pain, or discoloration after a traumatic injury should undergo dental radiography. Radiographs can show the degree of tooth displacement and indicate a root or bony fracture or permanent tooth bud displacement [33].

\section{Dental fractures}

\section{Infraction}

A tooth infraction is defined as an incomplete fracture or crack of the enamel, without a loss of tooth structure. Physical examination and radiographic findings are normal. No intervention is required other than reassurance. Complications are rare [34].

\section{Uncomplicated crown fracture}

An uncomplicated crown fracture is defined as a fracture to the enamel (or enamel and dentin) that does not involve the pulp. These are type 1 and 2 fractures in the Ellis classification system (Table 1). On physical examination, the yellow dentin may be visualized. Bleeding, significant pain, or pink, exposed pulp is not present. Urgent dental consultation is unnecessary for such injuries. Management consists of filing down rough edges of small fractures and attempting to restore tooth structure in larger fractures. Successful treatment depends on the degree of dentin exposure and the presence of concomitant injury to the periodontal ligament [34].

\section{Complicated crown fracture}

A complicated crown fracture, or Ellis type 3 fracture, involves the enamel, dentin, and pulp. Prompt dental care is required when pulp is exposed to prevent further injury and infection. In primary teeth, decisions on management are based

Table 1

Ellis classification of tooth fractures

\begin{tabular}{ll}
\hline Classification & Involvement \\
\hline Type 1 & Enamel only \\
Type 2 & Enamel and dentin \\
Type 3 & Enamel, dentin, and pulp \\
Type 4 & Root involvement \\
\hline
\end{tabular}


on the life expectancy of the tooth and on the degree of pulpal damage. Pulpal treatment options for permanent teeth include pulp capping and partial or complete pulpectomy [34]. Successful treatment depends on the duration of pulp exposure, the degree of dentin exposure, and the stage of root development.

\section{Root fracture}

Root fractures involve the cementum, dentin, and pulp. On clinical examination, the coronal fragment is mobile and attached to the gingiva, which may be displaced. Radiographic evidence of one or more horizontal fracture lines may be seen. Radiographic diagnosis may be difficult in a primary tooth if the succedaneous tooth is overlying. Primary teeth may be left in place if the fracture is in the apical third of the tooth and the crown segment is stable. However, if the fracture is in the middle or coronal third of the tooth, extraction is necessary because of the instability of the crown segment and the potential for infection from saliva contamination of the exposed pulp [35]. Treatment for permanent teeth with root fractures involves urgent repositioning and stabilization of the coronal fragment to allow healing of the periodontal ligament and neurovascular supply. The prognosis for permanent tooth root fractures is not affected by the location of the fracture. Pulp necrosis from displacement of the coronal segment occurs in $25 \%$ of patients with root fractures [34].

\section{Luxation injuries}

Luxation is displacement of a tooth from its normal position. Luxation injury to a permanent tooth is a true dental emergency, and immediate management is necessary to maintain the viability of an injured periodontal ligament.

\section{Subluxation}

Subluxation injury occurs when a tooth is abnormally loosened but not displaced, causing injury to the supporting periodontal ligament. On physical examination, the tooth is mobile and may exhibit sulcal bleeding. Radiographic study findings are normal. No intervention is required for primary teeth, other than dental follow-up. Permanent teeth should be stabilized and splinted as needed for comfort. The prognosis is good, but dental follow-up is necessary because pulpal necrosis may occur in cases in which the neurovascular supply is damaged [34].

\section{Lateral luxation}

Lateral luxation injury occurs when a tooth is displaced in a nonaxial direction, causing damage to the periodontal ligament and possible contusion or fracture of the supporting alveolar bone. On physical examination, the tooth is displaced laterally, with the crown usually directed palatally or lingually. The tooth may not be tender and is often immobile and trapped in its new position. Radiographic examination shows an increase in the periodontal ligament space and apical tooth displacement, with possible associated alveolar bone fracture. 
Primary teeth may be allowed to reposition passively or may be actively repositioned and splinted, unless the injury is severe or the life expectancy of the tooth is short. Permanent teeth require immediate active repositioning and splinting. Long-term risks include pulp necrosis and progressive root resorption [34].

\section{Intrusion}

Intrusion injury occurs when a tooth is driven apically and displaced into the alveolar bone, causing compression injury to the periodontal ligament and often fracturing the bony socket. On physical examination, the tooth appears shortened or, in severe cases, missing. It is immobile and nontender to the touch. Radiographic examination can visualize the apical displacement of the tooth as well as any disruption in the periodontal ligament. The treatment for intruded primary teeth is to allow spontaneous re-eruption, unless the tooth has been displaced into the succeeding permanent tooth. Permanent teeth may be repositioned actively or passively, depending on the degree of intrusion and maturity of the root apex. In permanent teeth with less mature roots, spontaneous re-eruption may be allowed. There is a high risk of pulp necrosis and root resorption in intrusion injuries to permanent teeth with mature root apices [34].

\section{Extrusion}

In extrusion injury, the tooth is displaced apically from the socket, resulting in damage to the periodontal ligament and the neurovascular supply. On physical examination, the tooth appears longer than adjacent teeth and is mobile. Radiographic examination shows a widening of the apical periodontal ligament space. Primary teeth may be extracted or repositioned. Permanent teeth should be urgently repositioned and stabilized to allow healing of the periodontal ligament and to maintain the integrity of the neurovascular supply. These patients must also be followed closely because they are at high risk for pulp necrosis [34].

\section{Avulsion injuries}

Tooth avulsion is the complete displacement of the tooth from its socket, severing the periodontal ligament. The prognosis for tooth survival is indirectly related to the time spent out of the oral cavity, with $85 \%$ to $97 \%$ survival at 5 minutes and near zero survival at 1 hour [36]. Avulsed primary teeth should not be replanted. Permanent teeth, however, should be replanted as soon as possible. The patient does not need to wait for professional medical assistance, and with proper guidance, the tooth can be replanted by the first capable person, such as the patient, a parent, or coach. The tooth should be handled by the crown to avoid damaging the periodontal ligament. Debris may be removed by gentle rinsing with saline or water, but scrubbing should be avoided. The tooth may then be replanted and held in place by biting down on a gauze pad (or by gentle finger pressure) until a splint can be placed by a medical or dental professional. If the tooth cannot be replanted within 5 minutes, it may be stored in a culture medium to preserve the vitality of periodontal ligament cells. The American Academy of 
Pediatric Dentistry recommends (in order of preference) the use of Viaspan, Hanks' balanced salt solution, cold milk, saliva, physiologic saline, or water. Milk is the preferred substance most readily available. The avulsed tooth may also be placed in a container of the child's saliva. Transport of the tooth in the child's mouth is not recommended because of risks of aspiration or further damage to the tooth. Close dental follow-up is necessary to evaluate for tooth survival. Tetanus prophylaxis and antibiotic coverage should be considered [34].

\section{Cervical lymphadenopathy}

Cervical lymphadenopathy is one of the most common clinical problems encountered in any pediatric primary care or emergency practice. Although cervical lymphadenopathy may herald the presence of a more serious systemic disease or malignancy, it is most often a normal and temporary response to a benign and localized infection. The dilemmas for the emergency physician are distinguishing between benign and severe causes of lymphadenopathy and initiating the proper investigation of the more perilous cases.

Lymphadenopathy is defined by the presence of nodal tissue measuring more than $1 \mathrm{~cm}$ in diameter [37]. This enlargement may be caused by proliferation of normal lymphocytes and macrophages in response to an antigen (eg, viral infection), infiltration of the lymph node by inflammatory cells in response to an infection of the node (lymphadenitis), proliferation within the lymph node of neoplastic lymphocytes or macrophages (lymphoma), or nodal infiltration with metabolite-loaded histiocytes in the storage diseases (eg, Gaucher and NiemanPick diseases).

The anterior cervical chain drains the mouth and pharynx and is the most common site of lymph node enlargement, resulting from common upper respiratory viral and bacterial infections. The posterior cervical chain and occipital nodes are most often enlarged in response to infection or dermatitis of the scalp. Generalized lymphadenopathy is defined as lymph node enlargement in two or more noncontiguous regions, including the liver and spleen. The discovery of enlarged cervical nodes should always prompt a complete examination of the remaining nodal regions to determine if it is a localized or systemic response.

\section{History and physical examination}

The differential diagnosis of cervical lymphadenopathy is quite broad (Box 1). The examination of the enlarged lymph nodes for tenderness, warmth, erythema, fluctuance, and mobility can yield clues to their cause. Most often, however, the associated findings on history and physical examination provide the salient information. A history of fever, rhinorrhea, sore throat, and cough or evidence of intraoral infection on physical examination are the most common findings in patients with cervical lymphadenopathy arising from an upper respiratory 


\section{Box 1. Differential diagnosis of cervical lymphadenopathy}

\section{Viral}

Rhinovirus, parainfluenza virus, influenzavirus, respiratory syncytial virus (RSV), coronavirus, adenovirus, Epstein-Barr virus (EBV), cytomegalovirus (CMV), rubella virus, rubeola, HSV, HIV, coxsackievirus

\section{Bacterial}

Staphylococcus and Streptococcus spp, anaerobes, Bartonella henselae, Mycobacterium tuberculosis

\section{Protozoal}

Toxoplasmosis

\section{Malignancies}

Leukemia, Iymphoma, neuroblastoma, rhabdomyosarcoma

\section{Other}

Kawasaki disease, collagen vascular disease

or intraoral infection. Historical factors that suggest the possibility of chronic diseases include malnutrition, weight loss, fever, and night sweats. Malignancy should be suspected in patients with anorexia, weight loss, or fevers and night sweats or lymphadenitis that is unresponsive to antibiotic treatment. Enlarged lymph nodes in patients with malignancies are usually nontender, firm, and rubbery and may be matted together. The presence of associated hepatosplenomegaly or bruises and petechiae also indicate potential malignancy.

\section{Differential diagnosis}

The most common cause of cervical lymphadenopathy is reactive intranodal cellular proliferation in response to an upper respiratory infection. Acute bilateral lymphadenitis is most often caused by the common upper respiratory tract viral infections, including rhinovirus, parainfluenza virus, influenza virus, RSV, coronavirus, and adenovirus [38]. Bilateral cervical lymphadenopathy is also found commonly in streptococcal pharyngitis and infectious mononucleosis. Unilateral suppurative lymphadenitis is more likely to be caused by a bacterial in- 
fection with Staph aureus, group A $\beta$-hemolytic streptococci, or other anaerobic organisms in patients with mucositis, gingival infections, or dental abscesses [38].

Viral infections that cause generalized lymphadenopathy, such as EBV and CMV may present initially with bilateral cervical lymphadenitis. Other causes of generalized lymphadenopathy include HIV, rubella virus, varicella-zoster virus, HSV, measles, and coxsackievirus [37].

Causes of subacute and chronic lymphadenopathy include $B$ henselae infection (cat-scratch disease), toxoplasmosis (most commonly presenting in the posterior chain), HIV, atypical mycobacteria, $M$ tuberculosis, and the nontuberculous mycobacterial infections.

Malignancy must always be considered when evaluating cervical lymphadenopathy. The cervical lymph nodes are the most common site of presentation of malignant tumors of the head and neck. In children ages 6 and younger, the most common malignant causes of enlarged cervical nodes include neuroblastoma, leukemia, rhabdomyosarcoma, and non-Hodgkin's lymphoma. In children older than 6 years, the most common causes are Hodgkin's lymphoma, non-Hodgkin's lymphoma, leukemia, and rhabdomyosarcoma.

Nonsuppurative cervical lymphadenopathy (with at least one node measuring greater than $1.5 \mathrm{~cm}$ ) is one of the five diagnostic criteria for Kawasaki disease. The others include bilateral bulbar conjunctival injection without exudates, erythematous mouth and pharynx, strawberry tongue with red, cracked lips, generalized erythematous rash, and changes in the peripheral extremities, consisting of either induration and erythema or desquamation. For the diagnosis, patients should have four of these five features as well as fever for at least 5 days' duration [39].

\section{Management}

In patients who have cervical lymphadenopathy and no physical findings or historical factors suggesting malignancy or serious systemic disease, observation with primary care follow-up is a reasonable care plan. Patients who have more worrisome findings warrant a more complete work-up. Initial evaluation should consist of a complete blood count with differential, erythrocyte sedimentation rate or C-reactive protein level, placement of a purified protein derivative tuberculin skin test, and plain chest radiography to evaluate for the presence of a mass, pulmonary disease, or mediastinal lymphadenopathy. Primary care or inpatient evaluation may also include serologic testing for EBV, CMV, and HIV infection. For patients who are suspected of having leukemias, pediatric hematologyoncology should be consulted to arrange bone marrow aspiration. Other testing may be guided by clinical suspicion, such as in cases of suspected mononucleosis or cat-scratch disease.

Patients who have a suspected bacterial lymphadenitis should be treated with oral antibiotics with staphylococcal and streptococcal coverage. Fine needle aspiration of a fluctuant node may be performed to guide antibiotic coverage. Patients who have a dominant lymph node of unknown cause that persists for 
more than 6 weeks or a lymph node that does not respond to antibiotic therapy require surgical referral for lymph node biopsy to rule out malignancy [40].

\section{References}

[1] American Academy of Pediatrics and American Academy of Family Physicians. Clinical practice guideline: diagnosis and management of acute otitis media. Pediatrics 2004;113:1451-65.

[2] Marx JA. Extenal otitis. In: Marx JA, editor. Rosen's emergency medicine: concepts and clinical practice. 5th edition. St. Louis (MO): Mosby; 2002. p. 932-3.

[3] Schwartz RH. Otitis externa. In: Long SS, editor. Principles and practice of pediatric infectious diseases. 2nd edition. Orlando (FL): Churchill Livingstone; 2003. p. 199-200.

[4] Eason JV. Otitis externa (PTG). In: Braunwald E, editor. Ferri's clinical advisor: instant diagnosis and treatment. St. Louis (MO): Mosby; 2005. p. 584-5.

[5] Paradise JL. Otitis media. In: Behrman RE, editor. Nelson textbook of pediatrics. 17th edition. Philadelphia: WB Saunders; 2004. p. 2138-49.

[6] Little P, Gould C, Williamson I, et al. Pragmatic randomized controlled trial of two prescribing strategies for childhood otitis media. BMJ 2001;322:336-42.

[7] Dowell SF, Butler JC, Giebink SG. Acute otitis media: management and surveillance in an era of pneumococcal resistance: a report from the Drug-Resistant Streptococcus pneumoniae Therapeutic Working Group. Pediatr Infect Dis J 1999;18:1-9.

[8] Dagan R, Hoberman A, Johnson C. Bacteriologic and clinical efficacy of high dose amoxicillin/ clavulanate in children with acute otitis media. Pediatr Infect Dis J 2001;20:829-37.

[9] Green SM, Rothrock SG. Single-dose intramuscular ceftriaxone for acute otitis media in children. Pediatrics 1993;91:23-30.

[10] Leibovitz E, Piglansky I, Raiz S, et al. Bacteriologic and clinical efficacy of one day vs. three day intramuscular ceftriaxone for treatment of nonresponsive acute otitis media in children. Pediatr Infect Dis J 2000;19:1040-5.

[11] Mantooth R, Hooker E. Foreign bodies, ear. Available at: http://www.emedicine.com/emerg/ topic185.htm. Accessed January 27, 2006.

[12] Barkin RM. Foreign body in the ear and nose. In: Barkin RM, editor. Pediatric emergency medicine: concepts and clinical practice. 2nd edition. St. Louis (MO): Mosby; 1997. p. 729-32.

[13] Palmer O. Button battery in the nose: an unusual foreign body. J Laryngol Otol 1994;108:871-2.

[14] Finkelstein JA. Oral ambu-bag insufflation to remove unilateral nasal foreign bodies. Am J Emerg Med 1996;14:57-8.

[15] Massick D, Tobin E. Otitis media. In: Cummings CW, editor. Otolaryngology: head and neck surgery. 4th edition. St. Louis (MO): Mosby; 2005. p. 4446-7.

[16] Gluckman W, Barricella R. Epistaxis. Available at: http://www.emedicine.com/ped/topic1618. htm. Accessed January 27, 2006.

[17] Evans J, Rothenhaus T. Epistaxis. Available at: http://master.emedicine.com/emerg/topic806. htm. Accessed January 27, 2006.

[18] Wald ER. Sinusitis. Pediatr Ann 1998;27(12):811-8.

[19] American Academy of Pediatrics. Appropriate use of antimicrobial agents. In: Pickering LK, editor. Red book: 2003 report of the Committee on Infectious Diseases. 26th edition. Elk Grove Village (IL): American Academy of Pediatrics; 2003. p. 696.

[20] Diament MJ. The diagnosis of sinusitis in infants and children: $x$-ray, computed tomography, and magnetic resonance imaging: diagnostic imaging of pediatric sinusitis. J Allergy Clin Immunol 1992;90(3 Pt 2):442-4.

[21] Wald ER, Chiponis D, Ledesma-Medina J. Comparative effectiveness of amoxicillin and amoxicillin-clavulanate potassium in acute paranasal sinus infections in children: a double-blind, placebo-controlled trial. Pediatrics 1986;77:795-800.

[22] Whitley RJ, Roizman B. Herpes simplex viral infection. Lancet 2001;357:1513-8. 
[23] Patel NJ, Sciubba J. Oral lesions in young children. Pediatr Clin North Am 2003;50(2):469-86.

[24] Rodu B, Mattingly G. Oral mucosal ulcers: diagnosis and management. J Am Dent Assoc 1992;123:83-6.

[25] American Academy of Pediatrics. Herpes simplex. In: Pickering LK, editor. Red book: 2003 report of the Committee on Infectious Diseases. 26th edition. Elk Grove Village (IL): American Academy of Pediatrics; 2003. p. 344-53.

[26] Amir J, Harel L, Smetana Z, et al. Treatment of herpes simplex gingivostomatitis with acyclovir in children: a randomized double-blind placebo controlled study. BMJ 1997;314:1800-3.

[27] Whitley RJ, Gnann JW. Acyclovir: a decade later. N Engl J Med 1992;327:782-9.

[28] Flores MT. Traumatic injuries in the primary dentition. Dent Traumatol 2002;18:287-98.

[29] Wilson S, Smith GA, Preisch J, et al. Epidemiology of dental trauma treated in an urban pediatric emergency department. Pediatr Emerg Care 1997;13:12.

[30] Kaste LM, Gift HC, Bhat M, et al. Prevalence of incisor trauma in persons 6-50 years of age: United States, 1988-1991. J Dent Res 1996;75:696-705.

[31] Jessee SA. Orofacial manifestations of child abuse and neglect. Am Fam Physician 1995; $52: 1829$

[32] Lane WG. Diagnosis and management of physical abuse in children. Clin Fam Pract 2003; 5(2):493-514.

[33] Nelson LP, Shusterman S. Emergency management of oral trauma in children. Curr Opin Pediatr 1997;9:242.

[34] American Academy of Pediatric Dentistry. American Academy of Pediatric Dentistry clinical guideline on management of acute dental trauma. Pediatr Dent 2004;26(7):120-7.

[35] Wilson CF. Management of trauma to primary and developing teeth. Dent Clin North Am 1995;39:133.

[36] Andreasen JO, Borum MK, Jacobsen HL, et al. Replantation of 400 avulsed permanent incisors: diagnosis of healing complications. Endod Dent Traumatol 1995;11:51-8.

[37] Leung AK, Robson WL. Childhood cervical lymphadenopathy. J Pediatr Health Care 2004;18(1):3-7.

[38] Peters TR, Edwards KM. Cervical lymphadenopathy and adenitis. Pediatr Rev 2000;21: $399-405$.

[39] American Academy of Pediatrics. Kawasaki syndrome. In: Pickering LK, editor. Red book: 2003 report of the Committee on Infectious Diseases. 26th edition. Elk Grove Village (IL): American Academy of Pediatrics; 2003. p. 392-5.

[40] Brown RL, Azizkhan RG. Pediatric head and neck lesions. Pediatr Clin North Am 1998;45: $889-905$. 